J. Chem. Eng. Data $2015 \quad$ Electronic Supporting Information

\title{
Melting points of potential liquid organic hydrogen carrier systems consisting of $\mathrm{N}$-alkylcarbazoles
}

\author{
Katharina Stark ${ }^{1}$, Philipp Keil ${ }^{1}$, Sebastian Schug ${ }^{1}$, Karsten Müller ${ }^{1, *}$, Peter Wasserscheid ${ }^{2}$, \\ Wolfgang Arlt ${ }^{1}$
}

1 Institute of Separation Science and Technology, Friedrich-Alexander-Universität Erlangen-Nürnberg, Egerlandstr. 3, 91058 Erlangen, Germany

2 Institute of Chemical Reaction Engineering, Friedrich-Alexander-Universität Erlangen-Nürnberg, Egerlandstr. 3, 91058 Erlangen, Germany

*Corresponding author, E-mail: karsten.mueller@fau.de (K. Müller)

\section{A. Conditions of the DSC measurements}

components/mixtures with $\mathrm{T}_{\mathrm{m}}<60^{\circ} \mathrm{C}$ :

1. Constant cooling rate with $\mathrm{dT} / \mathrm{dt}=+40 \mathrm{~K} / \mathrm{min}$ to the desired temperature

2. Isotherm segment for at least $\mathrm{t}=5 \mathrm{~min}$

3. Constant heating rate with $\mathrm{dT} / \mathrm{dt}=+10 \mathrm{~K} / \mathrm{min}$ to the desired temperature

4. Isotherm segment for $\mathrm{t}=5 \mathrm{~min}$

5. Steps 1-4 were repeated 2 times

components/mixtures with $\mathrm{T}_{\mathrm{m}}>60^{\circ} \mathrm{C}$

1. Constant heating rate with $\mathrm{dT} / \mathrm{dt}=+10 \mathrm{~K} / \mathrm{min}$ to the desired temperature

2. Isotherm segment for $\mathrm{t}=5 \mathrm{~min}$

3. Constant cooling rate with $\mathrm{dT} / \mathrm{dt}=+40 \mathrm{~K} / \mathrm{min}$ to the desired temperature

4. Isotherm segment for least $\mathrm{t}=5 \mathrm{~min}$

5. Steps 1-4 were repeated 2 times

If necessary the measurements were repeated with a lower heating rate ( 3 or $5 \mathrm{~K} / \mathrm{min}$ ) to obtain a higher resolution of the solid-liquid transition. Samples which did not crystallize during the DSC cooling segment, were only heated up once. 


\section{B. Experimental Data}

Table 1. Experimental SLE Data for the System N-ethylcarbazole (1) - N-ethyl-dodecahydro-carbazole (2) at Liquid Mole fraction $x$, Temperature $T$, and Pressure $p=0.1 \mathrm{MPa}^{a}$

\begin{tabular}{ccc}
\hline $\mathrm{x}_{1}$ & $\mathrm{~T} / \mathrm{K}$ & Solid Phase \\
\hline 0.10 & 285.6 & N-ethylcarbazole \\
0.12 & 290.0 & N-ethylcarbazole \\
0.14 & 294.6 & N-ethylcarbazole \\
0.18 & 299.0 & N-ethylcarbazole \\
0.23 & 303.3 & N-ethylcarbazole \\
0.28 & 307.3 & N-ethylcarbazole \\
0.35 & 312.7 & N-ethylcarbazole \\
0.44 & 316.7 & N-ethylcarbazole \\
0.53 & 321.7 & N-ethylcarbazole \\
0.66 & 325.0 & N-ethylcarbazole \\
0.76 & 328.5 & N-ethylcarbazole \\
0.88 & 334.3 & N-ethylcarbazole \\
1 & 342.4 & N-ethylcarbazole \\
\hline
\end{tabular}

${ }^{a}$ Standard uncertainties $\mathrm{u}$ are $\mathrm{u}\left(\mathrm{T}_{\mathrm{m}}\right)=1 \mathrm{~K}, \mathrm{u}(\mathrm{x})=0.02, \mathrm{u}(\mathrm{p})=5 \mathrm{kPa}$

Table 2. Experimental SLE Data for the System N-ethylcarbazole (1) - carbazole (2) at Liquid Mole fraction $x$, Melting Temperature $T_{m}$, Eutectic Temperature $T_{\text {eu }}$, and Pressure $p=0.1 \mathrm{MPa}^{a}$

\begin{tabular}{cccc}
\hline $\mathrm{x} 1$ & $\mathrm{~T}_{\mathrm{m}} / \mathrm{K}$ & $\mathrm{T}_{\mathrm{eu}} / \mathrm{K}$ & Solid Phase \\
\hline 0 & 517.1 & - & Carbazole \\
0.1 & 512.0 & 339.9 & Carbazole \\
0.2 & 500.9 & 340.1 & Carbazole \\
0.3 & 487.0 & 339.7 & Carbazole \\
0.4 & 477.5 & 340.0 & Carbazole \\
0.5 & 468.7 & 340.2 & Carbazole \\
0.6 & 457.1 & 340.0 & Carbazole \\
0.7 & 440.1 & 340.9 & Carbazole \\
0.8 & 418.7 & 340.6 & Carbazole \\
0.9 & 386.9 & 340.4 & Carbazole \\
1 & 342.4 & - & N-ethylcarbazole \\
\hline
\end{tabular}

${ }^{\mathrm{a} S t a n d a r d}$ uncertainties $\mathrm{u}$ are $\mathrm{u}\left(\mathrm{T}_{\mathrm{m}}\right)=1 \mathrm{~K}, \mathrm{u}\left(\mathrm{T}_{\text {eu }}\right)=2 \mathrm{Ku}(\mathrm{x})=0.001, \mathrm{u}(\mathrm{p})=5 \mathrm{kPa}$ 
Table 3. Experimental SLE Data for the System N-ethylcarbazole (1) - N-methylcarbazole (2) at Liquid Mole fraction $x$, Melting Temperature $T_{m}$, Eutectic Temperature $T_{\text {eu }}$, and Pressure $p=0.1 \mathrm{MPa}^{a}$

\begin{tabular}{cccc}
\hline $\mathrm{x} 1$ & $\mathrm{~T}_{\mathrm{m}} / \mathrm{K}$ & $\mathrm{T}_{\mathrm{eu}} / \mathrm{K}$ & Solid Phase \\
\hline 0 & 361 & - & N-methylcarbazole \\
0.249 & 344.1 & 315.9 & N-methylcarbazole \\
0.4 & 330.6 & 313.8 & N-methylcarbazole \\
0.5 & 321.4 & 314.1 & N-methylcarbazole \\
0.7 & 323.9 & 315.7 & N-ethylcarbazole \\
0.892 & 336.4 & 315.1 & N-ethylcarbazole \\
1 & 342.4 & - & N-ethylcarbazole \\
\hline
\end{tabular}

${ }^{\mathrm{a}}$ Standard uncertainties $\mathrm{u}$ are $\mathrm{u}\left(\mathrm{T}_{\mathrm{m}}\right)=1 \mathrm{~K}, \mathrm{u}\left(\mathrm{T}_{\text {eu }}\right)=2 \mathrm{~K}, \mathrm{u}(\mathrm{x})=0.001, \mathrm{u}(\mathrm{p})=5 \mathrm{kPa}$

Table 4. Experimental SLE Data for the System N-ethylcarbazole (1) - N-propylcarbazole (2) at Liquid Mole fraction $x$, Melting Temperature $T_{m}$, Eutectic Temperature $T_{e u}$, and Pressure $p=0.1 \mathrm{MPa}^{a}$

\begin{tabular}{cccc}
\hline $\mathrm{x} 1$ & $\mathrm{~T}_{\mathrm{m}} / \mathrm{K}$ & $\mathrm{T}_{\mathrm{eu}} / \mathrm{K}$ & Solid Phase \\
\hline 0 & 320.3 & - & N-propylcarbazole \\
0.052 & 319.9 & - & N-propylcarbazole \\
0.1 & 318 & 294.7 & N-propylcarbazole \\
0.2 & 312.8 & 295.9 & N-propylcarbazole \\
0.3 & 306.8 & 291.9 & N-propylcarbazole \\
0.4 & & 291.9 & N-propylcarbazole \\
0.494 & & 296.1 & N-ethylcarbazole \\
0.498 & & 294.5 & N-ethylcarbazole \\
0.548 & & 298.0 & N-ethylcarbazole \\
0.601 & 316.8 & 299.9 & N-ethylcarbazole \\
0.7 & 321.3 & 299.6 & N-ethylcarbazole \\
0.8 & 329.1 & 298.9 & N-ethylcarbazole \\
0.899 & 336.4 & 297.3 & N-ethylcarbazole \\
1 & 342.4 & - & N-ethylcarbazole \\
\hline
\end{tabular}

${ }^{\mathrm{a}}$ Standard uncertainties $\mathrm{u}$ are $\mathrm{u}\left(\mathrm{T}_{\mathrm{m}}\right)=1 \mathrm{~K}, \mathrm{u}\left(\mathrm{T}_{\text {eu }}\right)=2 \mathrm{~K}, \mathrm{u}(\mathrm{x})=0.001, \mathrm{u}(\mathrm{p})=5 \mathrm{kPa}$ 
Table 5. Experimental SLE Data for the System N-ethylcarbazole (1) $-\mathrm{N}$-isopropylcarbazole (2) at Liquid Mole fraction $x$, Melting Temperature $T_{m}$, Eutectic Temperature $T_{\text {eu }}$, and Pressure $p=0.1 \mathrm{MPa}^{a}$

\begin{tabular}{cccc}
\hline $\mathrm{x} 1$ & $\mathrm{~T}_{\mathrm{m}} / \mathrm{K}$ & $\mathrm{T}_{\mathrm{eu}} / \mathrm{K}$ & Solid Phase \\
\hline 0 & 393.9 & - & $\mathrm{N}$-isopropylcarbazole \\
0.1 & 393.2 & 373.2 & $\mathrm{~N}$-isopropylcarbazole \\
0.2 & 393.2 & 370.2 & $\mathrm{~N}$-isopropylcarbazole \\
0.3 & 391.3 & 370.2 & $\mathrm{~N}$-isopropylcarbazole \\
0.4 & 387.3 & 367.4 & $\mathrm{~N}$-isopropylcarbazole \\
0.45 & 384.2 & 366.4 & $\mathrm{~N}$-isopropylcarbazole \\
0.5 & 382.2 & 335.5 & $\mathrm{~N}$-isopropylcarbazole \\
0.55 & 378.8 & 333.1 & $1: 1$ - compound \\
0.6 & 378.2 & 334.1 & $1: 1$ - compound \\
0.7 & 371.9 & 337.2 & $1: 1$ - compound \\
0.8 & 364.3 & 337.9 & $1: 1$ - compound \\
0.9 & 351.6 & 338.1 & $1: 1$ - compound \\
0.95 & 342 & 338.2 & $1: 1$ - compound \\
1 & 342.4 & - & $\mathrm{N}$-ethylcarbazole \\
\hline
\end{tabular}

${ }^{\mathrm{a}}$ Standard uncertainties $\mathrm{u}$ are $\mathrm{u}\left(\mathrm{T}_{\mathrm{m}}\right)=1 \mathrm{~K}, \mathrm{u}\left(\mathrm{T}_{\text {eu }}\right)=2 \mathrm{~K}, \mathrm{u}(\mathrm{x})=0.001, \mathrm{u}(\mathrm{p})=5 \mathrm{kPa}$

Table 6. Experimental SLE Data for the System N-ethylcarbazole (1) - N-butylcarbazole (2) at Liquid Mole fraction $x$, Melting Temperature $T_{m}$, Eutectic Temperature $T_{e u}$, and Pressure $p=0.1 \mathrm{MPa}^{a}$

\begin{tabular}{cccc}
\hline $\mathrm{x} 1$ & $\mathrm{~T}_{\mathrm{m}} / \mathrm{K}$ & $\mathrm{T}_{\text {eu }} / \mathrm{K}$ & Solid Phase \\
\hline 0 & 330.6 & - & N-butylcarbazole \\
0.2 & 322.2 & 303.9 & N-butylcarbazole \\
0.325 & 316.2 & 302.3 & N-butylcarbazole \\
0.51 & & 304.2 & N-ethylcarbazole \\
0.66 & 319.8 & 303.9 & N-ethylcarbazole \\
0.91 & 338 & 304.5 & N-ethylcarbazole \\
1 & 342.4 & - & N-ethylcarbazole \\
\hline
\end{tabular}

${ }^{\mathrm{a}}$ Standard uncertainties $\mathrm{u}$ are $\mathrm{u}\left(\mathrm{T}_{\mathrm{m}}\right)=1 \mathrm{~K}, \mathrm{u}\left(\mathrm{T}_{\mathrm{eu}}\right)=2 \mathrm{~K}, \mathrm{u}(\mathrm{x})=0.001, \mathrm{u}(\mathrm{p})=5 \mathrm{kPa}$ 
Table 7. Experimental SLE Data for the System N-propylcarbazole (1) - N-butylcarbazole (2) at Liquid Mole fraction $x$, Melting Temperature $T_{m}$, Eutectic Temperature $T_{\text {eu }}$, Temperature of Transition $T_{t r}$ and Pressure $\mathrm{p}=0.1 \mathrm{MPa}^{\mathrm{a}}$

\begin{tabular}{ccccc}
\hline $\mathrm{x} 1$ & $\mathrm{~T}_{\mathrm{m}} / \mathrm{K}$ & $\mathrm{T}_{\mathrm{eu}} / \mathrm{K}$ & $\mathrm{T}_{\mathrm{tr}} / \mathrm{s}$ & Solid Phase \\
\hline 0 & 330.6 & & & N-butylcarbazole \\
0.1 & 328.1 & 295.7 & & $\mathrm{~N}$-butylcarbazole \\
0.2 & 324.6 & 296.5 & & $\mathrm{~N}$-butylcarbazole \\
0.3 & 318.1 & 299.9 & & $\mathrm{~N}$-butylcarbazole \\
0.4 & 311 & 297.5 & & $\mathrm{~N}$-butylcarbazole \\
0.48 & 306 & 297.8 & 284.3 & $\mathrm{~N}$-butylcarbazole \\
0.50 & 305.3 & 297.2 & 285.6 & $\mathrm{~N}$-butylcarbazole \\
0.53 & 303.9 & 297.9 & 284.9 & $\mathrm{~N}$-butylcarbazole \\
0.56 & 300.1 & - & 285.3 & $\mathrm{~N}$-butylcarbazole \\
0.6 & 299.7 & 296.5 & 284.8 & $\mathrm{~N}$-propylcarbazole \\
0.7 & 308.5 & 298.4 & 283.2 & $\mathrm{~N}$-propylcarbazole \\
0.8 & 314.1 & 299.5 & 282.8 & $\mathrm{~N}$-propylcarbazole \\
0.9 & 317.8 & 298.2 & 281.9 & $\mathrm{~N}$-propylcarbazole \\
1 & 320.3 & & & $\mathrm{~N}$-propylcarbazole \\
\hline
\end{tabular}

${ }^{a}$ Standard uncertainties $\mathrm{u}$ are $\mathrm{u}\left(\mathrm{T}_{\mathrm{m}}\right)=1 \mathrm{~K}, \mathrm{u}\left(\mathrm{T}_{\text {eu }}\right)=2 \mathrm{~K}, \mathrm{u}\left(\mathrm{T}_{\mathrm{TR}}\right)=2 \mathrm{~K}, \mathrm{u}(\mathrm{x})=0.001, \mathrm{u}(\mathrm{p})=5 \mathrm{kPa}$ 


\section{NMR spectra of synthesized compounds}

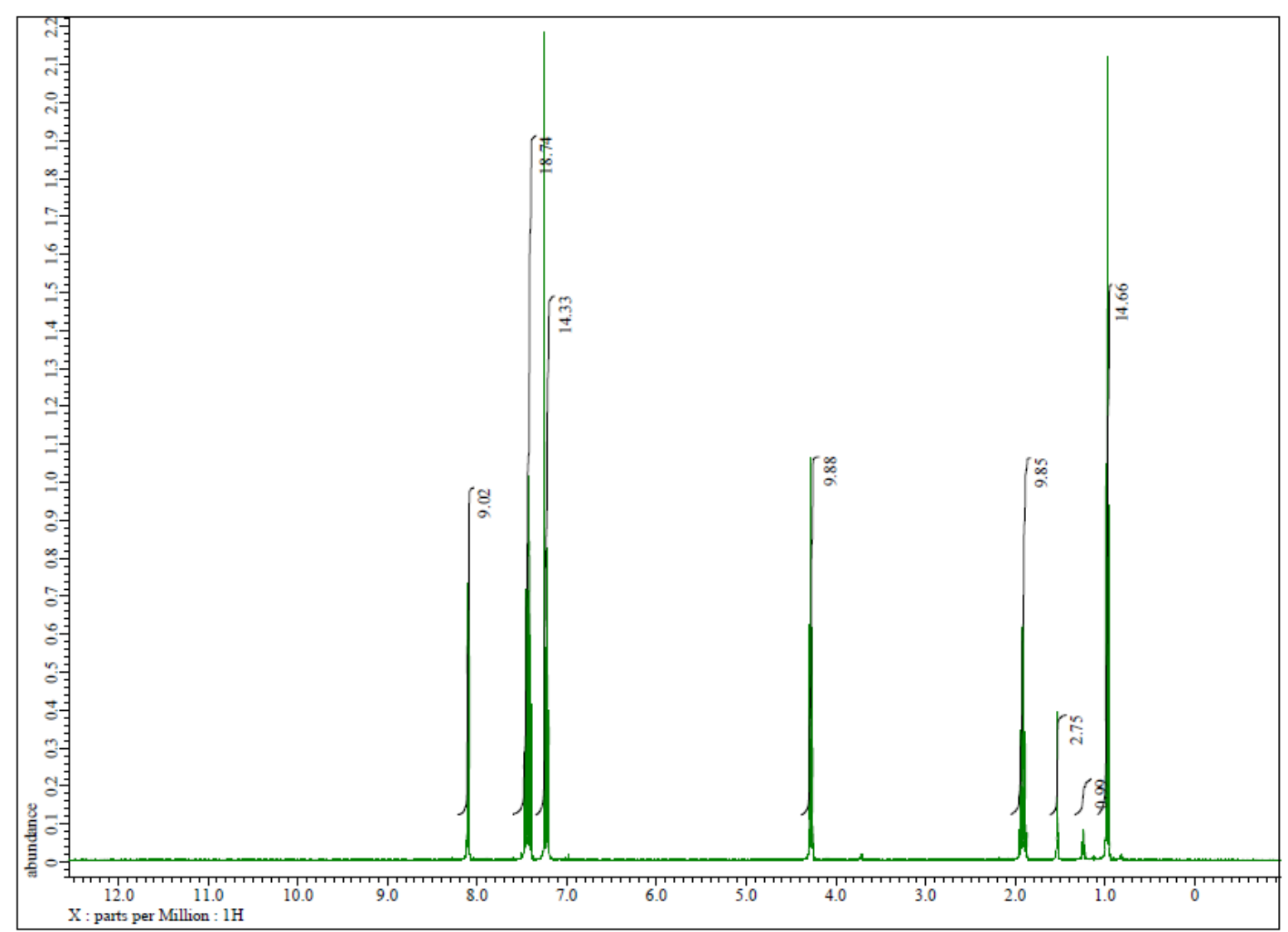

Figure 1: $1 \mathrm{H}$ NMR spectrum of N-propylcarbazole 


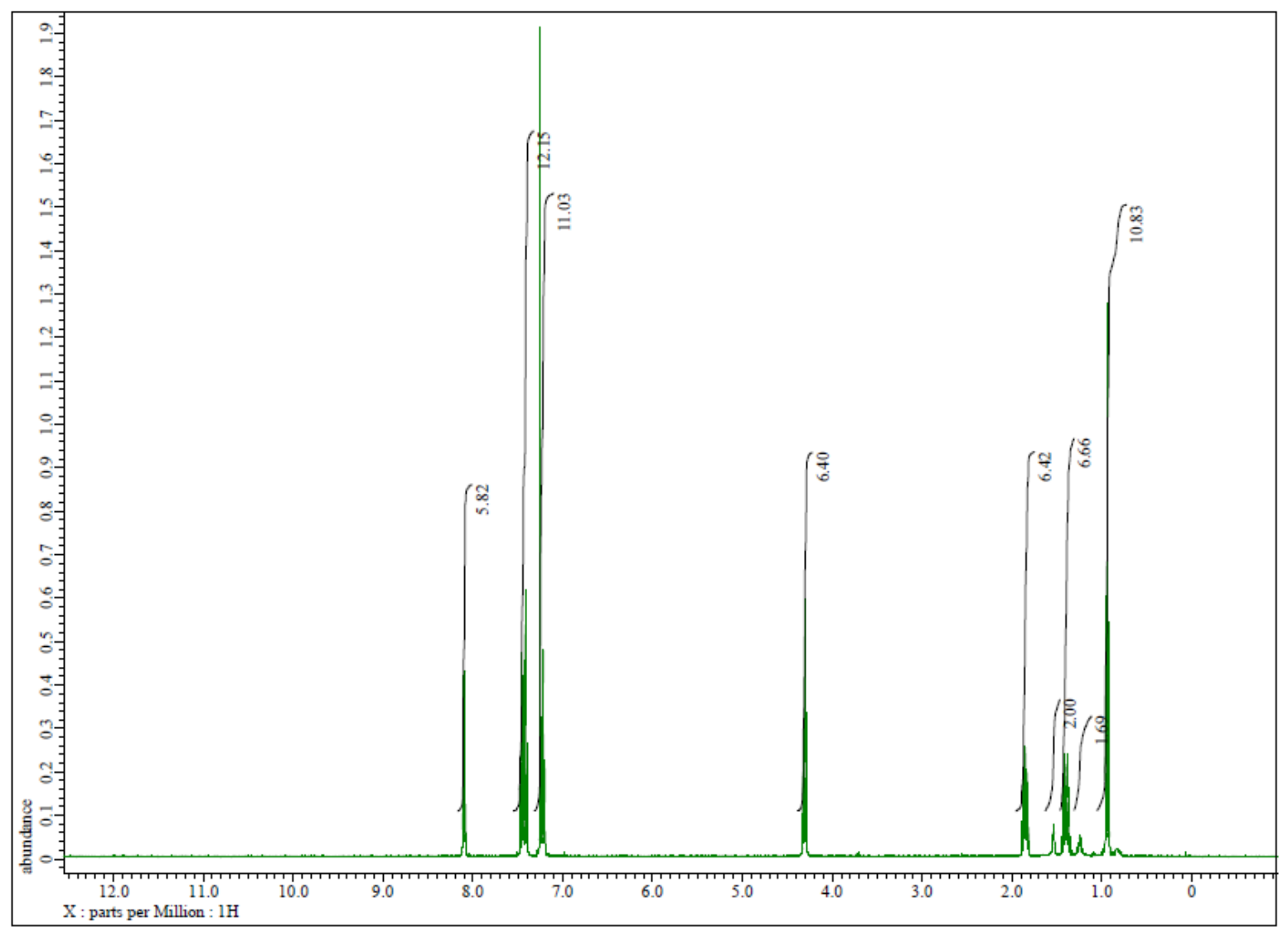

Figure 2. 1H NMR spectrum of N-butylcarbazole 


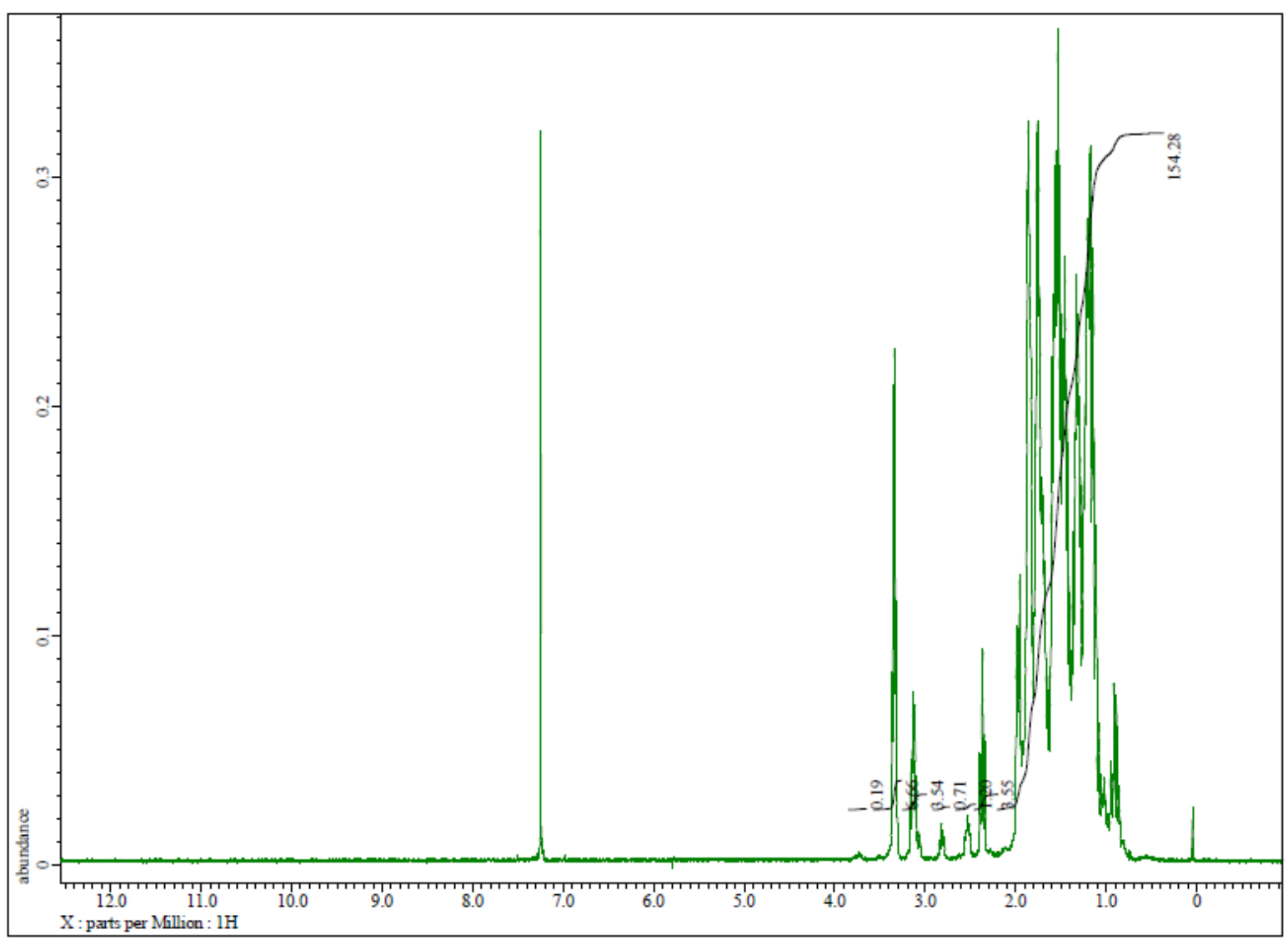

Figure 3. $1 \mathrm{H}$ NMR spectrum of $12 \mathrm{H}$-carbazole 